九州大学学術情報リポジトリ

Kyushu University Institutional Repository

\title{
Revealing the Formation Mechanism of Alloyed Pd-Ru Nanoparticles: A Conversion Measurement Approach Utilizing a Microflow Reactor
}

\section{Asano, Shusaku}

Department of Chemical Engineering, Kyoto University

Maki, Taisuke

Department of Chemical Engineering, Kyoto University

Sebastian, Victor

Department of Chemical \& Environmental Engineering, Aragon Institute of Nanoscience (INA), University of Zaragoza

Jensen, Klavs F.

Department of Chemical Engineering, Massachusetts Institute of Technology

他

ht tp://hdl. handle. net/2324/2235385

出版情報 : Langmuir. 35 (6)，pp.2236-2243，2019-02-12. American Chemical Society バージョン：

権利関係 : 


\section{Revealing the formation mechanism of alloyed Pd-}

\section{Ru nanoparticles: a conversion measurement}

\section{approach utilizing a microflow reactor}

Shusaku Asano, ${ }^{*}{ }^{\dagger, \diamond}$ Taisuke Maki ${ }^{\dagger}$ Victor Sebastian, ${ }^{\ddagger, \S}$ Klavs F. Jensen ${ }^{\#}$, and Kazuhiro Mae ${ }^{\dagger}$

${ }^{\dagger}$ Department of Chemical Engineering, Kyoto University, Kyoto 6158510, Japan

${ }^{\ddagger}$ Department of Chemical \& Environmental Engineering, Aragon Institute of Nanoscience (INA), University of Zaragoza, Campus Rio Ebro, 50018 Zaragoza, Spain

${ }^{\S}$ CIBER de Bioingeniería, Biomateriales y Nanomedicina (CIBER-BBN), Centro de Investigación Biomédica en Red, C/Monforte de Lemos 3-5, Pabellón 11, 28029 Madrid, Spain

\# Department of Chemical Engineering, Massachusetts Institute of Technology, 77

Massachusetts Avenue, Cambridge, MA 02139, USA 
ABSTRACT: The synthesis of alloyed nanoparticles has been studied extensively; however, the formation mechanisms involved remain unclear. Here, we reveal the detailed formation mechanism of alloyed nanoparticles in a Pd-Ru system using a semi-batch polyol method in which the simultaneous rapid reduction of both precursors was assumed to be the critical mechanism. We employed a microflow reactor to realize rapid heating and cooling. A significant difference in the reaction rate between the two precursors was observed. Pd was reduced in seconds, but the reduction of Ru was two orders of magnitude slower than that of Pd and was not as rapid as previously assumed. Further investigation of the semi-batch method was performed to trace changes in the particle sizes and composition. Through quantitative and multilateral evidence, we concluded that the formation of low-crystallinity seeds, followed by solid state diffusion, is the governing mechanism for the formation of alloyed Pd-Ru nanoparticles.

KEYWORDS: rapid heating, polyol method, solid solution, solid state diffusion, reaction kinetics 


\section{INTRODUCTION}

Bimetallic nanoparticles are vital for practical performance in catalysis, gas storage, and sensing applications ${ }^{1}$. There are four possible architectures in a bimetallic system: a solid solution alloy, a core-shell structure, a heteroaggregate, and a mixture of monometallic nanoparticles ${ }^{2}$. These architectures drastically change the nanoparticle characteristics and properties. For example, Alayoglu and Eichhorn examined the catalytic activity during CO oxidation with a Rh@Pt coreshell structure, a Pt-Rh alloy, and a Pt+Rh mixture, and they found that the temperature at which CO was oxidized differed considerably, ranging from $70^{\circ} \mathrm{C}$ for Rh@Pt to $160^{\circ} \mathrm{C}$ for the Pt+Rh mixture ${ }^{3}$. The influence of the bimetallic structure on the catalytic activity is based on lattice shrinking/expansion generated by the atomic size difference, adsorption sites on the particle surface, and elementary reaction steps with metal and adsorbed species ${ }^{4-6}$. Importantly, metals with a low affinity that segregate spontaneously in the bulk phase may form a nanoscale alloy. Synthetic methods for bimetallic nanostructures have been widely studied and developed to provide desirable architectures with a selected combination of elements ${ }^{7-9}$. Compared with monometallic nanoparticles, the synthesis of bimetallic nanoparticles is far more difficult and complicated. Many factors of each element, such as crystal structure, bonding strength, surface energy, atomic size, and electron vacancy, can interact and influence the nucleation and growth processes $^{10}$.

To synthesize alloyed nanoparticles, coreduction, in which two metal precursors are reduced in a one-pot system, is the easiest and most common method ${ }^{11-13}$. However, an interaction between precursors and the reaction kinetics often prevent alloying in the coreduction method. For example, the coreduction of $\mathrm{PdCl}_{2}$ and $\mathrm{HAuCl}_{4}$ in ethanol-water in the presence of poly(N-vinyl-2- 
pyrrolidone) (PVP) at $30^{\circ} \mathrm{C}$ resulted in a Au@Pd core-shell structure ${ }^{14}$. $\mathrm{AuCl}_{4}{ }^{-}$oxidizes reduced $\operatorname{Pd}(0)$; therefore, $\operatorname{Pd}(0)$ can be stable only after all Au species are reduced. The mechanism was revealed by tracing the concentration profile of the precursor during reduction by UV-Vis spectroscopy ${ }^{14}$.

Although a rate analysis of the precursor reduction provides insight into the evolution mechanism of various nanostructures, precursor conversion is rarely measured because of challenges faced in separating the product nanoparticles from the unreacted precursors. Recently, Wang et al. avoided the separation problem by conducting experiments without a capping agent ${ }^{15}$. The synthesized particles spontaneously aggregated and separated rapidly from the solution. The authors successfully obtained kinetics information about the reduction of $\mathrm{PdCl}_{4}{ }^{2-}$ in polyol. They found that the twin structure and shape of Pd nanocrystals can be controlled quantitatively by the reaction rate of the precursor reduction. Because the reaction temperature in their experiment was high, reaching $160^{\circ} \mathrm{C}$, the reaction time was set to a minimum of $25 \mathrm{~s}$. This shorter reaction time would cause not only difficulty in handling but also unreliable results due to the time delay in mixing and heating.

Here, we hypothesized that a conversion measurement utilizing a microflow reactor would expand our understanding of the mechanisms of bimetallic nanostructure formation. A microflow reactor has advantages in mass and heat transfer because of the short diffusion length and the high surfaceto-volume ratio ${ }^{16}$. Enhanced mass transfer provides an ideal homogeneous reaction condition for particle synthesis. Tofighi et al. utilized a microflow reactor for synthesizing monodispersed AuPd nanoparticles with a size of approximately $1 \mathrm{~nm}$ by achieving a mixing time of approximately 2 $\mathrm{ms}^{17}$. An in situ study of gold nanoparticle synthesis was also conducted using the same device 
and X-ray absorption spectroscopy to show the potential of microflow reactors in the nanoparticle study ${ }^{18}$. A microflow reactor also has advantages for conducting reactions at elevated temperatures and pressures. A back-pressure regulator can comfortably and safely create pressurized reaction conditions in the flow scheme. For instance, various metallic nanostructures have been produced in a remarkably short time, down to tens of seconds, through rapid heating in a single pressurized microflow reactor ${ }^{19}$. Microchips made of silicon carbide yield the best performance in these applications ${ }^{20,21}$; however, commercial miniature tubing is becoming increasingly popular because of its low cost. Commercially available tubes and fittings also show excellent performance, displaying characteristics of submillisecond mixing ${ }^{22}$, a uniform residence time distribution ${ }^{23,24}$, and a heat transfer coefficient greater than $20,000 \mathrm{~W} / \mathrm{m}^{2} \mathrm{~K}$ when the materials are appropriately chosen and configured ${ }^{25}$. Microflow reactors are now widely used for the fundamental study of nanoparticles and colloids ${ }^{26-28}$, as well as in industrial continuous crystallization plants, at the pilot scale $^{29}$.

We selected the synthesis of Pd-Ru alloyed nanoparticles (with a semi-batch polyol method) as the model case for our study. Palladium and ruthenium have a low affinity for one another, different crystalline structures, and melting points that differ by $700 \mathrm{~K}^{1}$; therefore, they spontaneously separate in the bulk phase ${ }^{30}$. Kusada et al. ${ }^{31}$ first reported the method for making solid solution alloy nanoparticles in this system. They slowly injected an aqueous solution containing $\mathrm{RuCl}_{3}$ and $\mathrm{K}_{2} \mathrm{PdCl}_{4}$ into triethylene glycol (TEG) containing PVP that was heated to $200^{\circ}$ C. They employed a semi-batch system, aiming to realize the simultaneous and instant reduction of both precursors. They reported that the Pd ions were reduced at approximately $90^{\circ} \mathrm{C}$, and the Ru ions were reduced at approximately $160{ }^{\circ} \mathrm{C}$ by TEG; thus, a common batch method resulted in phase separation that was attributed to a sequential reduction during heating. The 
alloyed Pd-Ru nanoparticle showed synergetically enhanced catalytic activity for CO oxidation, the Suzuki-Miyaura cross-coupling reaction ${ }^{32}, \mathrm{NO}_{\mathrm{x}}$ reduction ${ }^{33}$, and formic acid electrooxidation ${ }^{34}$.

In this work, we report the results of the conversion measurement of $\mathrm{PdCl}_{4}{ }^{2-}$ and $\mathrm{Ru}^{3+}$ in TEG at $200^{\circ} \mathrm{C}$ using a microflow reactor. The reaction time was precisely controlled from several seconds to minutes. The required time for reduction differed by orders of magnitude between the precursors. Experiments using the microflow reactor, combined with the original semi-batch method and the collection of multiple samples during operation, revealed the critical role of solid state diffusion in forming alloyed nanoparticles.

\section{EXPERIMENTAL SECTION}

$\mathrm{Na}_{2} \mathrm{PdCl}_{4}$ (Inuisho Precious Metals, Osaka, Japan), $\mathrm{RuCl}_{3} \cdot n \mathrm{H}_{2} \mathrm{O}$ (Wako Pure Chemical Industries, Osaka, Japan), TEG (Alfa Aesar, Ward Hill, MA, USA), PVP (K30, Wako), Y( $\left(\mathrm{NO}_{3}\right)_{3} \cdot 6 \mathrm{H}_{2} \mathrm{O}$ (Aldrich, St. Louis, MO, USA), and hydrochloric acid (Wako) were purchased and used without further purification. Ultra-purified water was prepared using a Milli-Q water purification system and used throughout the experiment. Commercial $\mathrm{RuCl}_{3} \cdot n \mathrm{H}_{2} \mathrm{O}$ has been reported to contain $\mathrm{Ru}^{\mathrm{IV}}$ hydrolysates and polymers ${ }^{35}$; nevertheless, we assumed the composition to be $\mathrm{RuCl}_{3} \cdot 3 \mathrm{H}_{2} \mathrm{O}$ in this study, according to Kusada et al. ${ }^{31}$.

Conversion measurement of precursors using a microflow reactor. Figure 1(a) shows the reactor system. A $1 \mathrm{mM}$ solution of the metal precursor in TEG was prepared immediately before the experiment. Perfluoroalkoxy alkane (PFA) tubing was used as a reactor and placed in an oil bath set at $200^{\circ} \mathrm{C}$. For short residence times down to $1 \mathrm{~s}$, 1-m-long tubing, with an i.d. of $0.5 \mathrm{~mm}$ was used. For residence times longer than 20 s, 2.67-m-long tubing, with an i.d. of $1.0 \mathrm{~mm}$ was 
used. A tube-in-tube heat exchanger was connected to the reactor to cool the reaction solution immediately. PFA tubing, with an i.d. of $0.5 \mathrm{~mm}$ and an o.d. of $0.7 \mathrm{~mm}$, was used as the inner tube. PFA tubing, with an i.d. of $1.0 \mathrm{~mm}$ and an o.d. of 1/16”, was used as the outer tube. Water cooled with ice was circulated through the outer tube at a flow rate of $30-70 \mathrm{~mL} \mathrm{~min}{ }^{-1}$. The reaction solution was fed to the reactor through a syringe pump (PHD Ultra, Harvard Apparatus, Holliston, MA, USA). Samples were collected at the outlet of the inner tube of the heat exchanger.

Conversion measurements of $\mathrm{Na}_{2} \mathrm{PdCl}_{4}$ were conducted according to the method developed by Wang et al. ${ }^{15} \mathrm{~A} 1 \mathrm{~mL}$ sample was diluted with $9 \mathrm{~mL}$ of a $0.1 \mathrm{M} \mathrm{HCl}$ aqueous solution to prevent further reaction. Then, the diluted sample was centrifuged for 10 minutes at 10,000 rpm (H-1500F, Kokusan, Tokyo, Japan). The remaining amount of $\mathrm{PdCl}_{4}{ }^{2-}$ in the supernatant was determined through UV-Vis spectroscopy (Multispec-1500, Shimadzu, Kyoto, Japan) using the characteristic absorbance peak at $279 \mathrm{~nm}$.

For the conversion measurement of $\mathrm{RuCl}_{3}, 1 \mathrm{~mL}$ of sample was diluted with $19 \mathrm{~mL}$ of an aqueous solution containing $0.1 \mathrm{M} \mathrm{HCl}$ and $1 \mathrm{mM}$ of $\mathrm{Y}\left(\mathrm{NO}_{3}\right)_{3}$. $\mathrm{HCl}$ was added to preserve the precursor, and $\mathrm{Y}\left(\mathrm{NO}_{3}\right)_{3}$ was added as the internal standard. Then, the diluted sample was centrifuged for 10 minutes at 10,000 rpm. The remaining metal ion content in the supernatant was determined through inductively coupled plasma atomic emission spectroscopy (ICP-AES) with an ICPS-7500 (Shimadzu).

When both $\mathrm{Na}_{2} \mathrm{PdCl}_{4}$ and $\mathrm{RuCl}_{3}$ were added to the reactant solution to investigate the interaction between precursors, the amount of both elements dissolved in the product solution was quantified through the ICPS-7500. 
Flow synthesis of Pd-Ru nanoparticles with rapid heating. Figure 1(b) illustrates the reactor system. A reactant solution was prepared by mixing $5 \mathrm{~mL}$ of a precursor solution and $50 \mathrm{~mL}$ of a reductant solution. The precursor solution contained $12.5 \mathrm{mM}$ of $\mathrm{RuCl}_{3}$ and $\mathrm{K}_{2} \mathrm{PdCl}_{4}$ in water. The reductant solution contained $40 \mathrm{mM}$ of PVP on a monomer basis in TEG. Two stainless steel tubes were connected and used as a reactor. The first tube was $7 \mathrm{~cm}$ long, with an i.d. of $0.1 \mathrm{~mm}$. The second tube was $72 \mathrm{~cm}$ long, with an i.d. of $1.0 \mathrm{~mm}$. The reactor was placed in an oil bath at $200^{\circ} \mathrm{C}$. Extra tubing for cooling, with an i.d. of $0.5 \mathrm{~mm}$, was connected to the end of the reactor and placed in a water bath that was cooled with ice. A back-pressure regulator (BPR, IDEX Health \& Science, Oak Harbor, WA, USA) set to $250 \mathrm{psi}$ (1.7 MPa) was placed at the outlet to prevent boiling. The reactant solution was fed to the reactor through a syringe pump (PHD Ultra, Harvard Apparatus) at $5.5 \mathrm{~mL} \mathrm{~min}^{-1}$. The heating time in this system was estimated to be $0.3 \pm 0.1 \mathrm{~s}$ (see Supporting Information).

Semi-batch synthesis of alloyed Pd-Ru nanoparticles through the simultaneous addition of precursors. Figure 1(c) shows the reactor system. Semi-batch synthesis was performed according to the original method by Kusada et al. ${ }^{31}$, except for the injection time, which was not specified in the original paper. A reductant solution was prepared by dissolving $444 \mathrm{mg}$ ( $4 \mathrm{mmol}$ on a monomer basis) of PVP into $100 \mathrm{~mL}$ of TEG. A precursor solution was prepared by dissolving $147.3 \mathrm{mg}$ (0.5 mmol) of $\mathrm{Na}_{2} \mathrm{PdCl}_{4}$ and $131.1 \mathrm{mg}(0.5 \mathrm{mmol})$ of $\mathrm{RuCl}_{3} \cdot n \mathrm{H}_{2} \mathrm{O}$ into $40 \mathrm{~mL}$ of water. The reductant solution was heated to $200^{\circ} \mathrm{C}$ in a round-bottomed flask using a heating mantle (GB-3, Taika, Osaka, Japan). The solution was stirred at approximately $200 \mathrm{rpm}$ using an overhead stirrer. The precursor solution was injected into the reductant solution over a 25-minute period through a syringe pump (PHD Ultra, Harvard Apparatus). The solution was kept at $200^{\circ} \mathrm{C}$ for 275 minutes and stirred after the injection was completed. 
The samples for transmission electron microscopy (TEM) and X-ray powder diffraction (XRD) were prepared separately. For TEM analysis, a total of $0.05 \mathrm{~mL}$ of the reactant solution was sampled during the reaction. The solution was diluted in $1 \mathrm{~mL}$ of methanol for quenching and then dropped immediately onto a carbon-coated copper grid. Because XRD analysis requires an isolated sample in tens of milligrams, the entire solution from a batch was used to prepare a sample. At a certain reaction time, the injection was stopped, and the entire reactant solution was poured into a stainless steel container placed in an ice bath for immediate quenching. After quenching, the remaining glycol and ions were replaced in the water using a dialysis membrane with a molecular weight cutoff of $50 \mathrm{kD}$ (Float-A-Lyzer, Repligen, Waltham, MA, USA). Next, the dialyzed solution was concentrated by gently evaporating the water. Particles were then separated and washed with methanol by repeating the 1-hour centrifugation twice at 12,000 rpm.

\section{Semi-batch synthesis of Pd-Ru nanoparticles with the sequential addition of precursors. Two}

precursor solutions were prepared separately by dissolving $73.7 \mathrm{mg}(0.25 \mathrm{mmol})$ of $\mathrm{Na}_{2} \mathrm{PdCl}_{4}$ and $65.6 \mathrm{mg}(0.25 \mathrm{mM})$ of $\mathrm{RuCl}_{3} \cdot n \mathrm{H}_{2} \mathrm{O}$ into $5 \mathrm{~mL}$ of TEG. A reductant solution was prepared by dissolving $222 \mathrm{mg}$ ( $2 \mathrm{mmol}$ on a monomer basis) of PVP into $50 \mathrm{~mL}$ of TEG. The reductant solution was heated to $200^{\circ} \mathrm{C}$ in a round-bottomed flask using a heating mantle. The solution was stirred at approximately $200 \mathrm{rpm}$ using an overhead stirrer. One precursor solution was injected into the reductant solution over 20 minutes through a syringe pump. After 5 minutes of aging, another precursor solution was injected over 20 minutes. The solution was kept at $200^{\circ} \mathrm{C}$ while stirring for 45 minutes after completing the injection. A total of $0.05 \mathrm{~mL}$ of the reactant solution was sampled after each aging time. The sample was diluted into $1 \mathrm{~mL}$ of methanol and dropped onto a carbon-coated copper grid for subsequent TEM analysis. Samples for the XRD analysis were separately synthesized following the same reported procedure and isolated by centrifugation 
for 1 hour at 12,000 rpm. Pd seeds and Ru seeds were also prepared as XRD standards and isolated by centrifugation for 1 hour at 12,000 rpm.
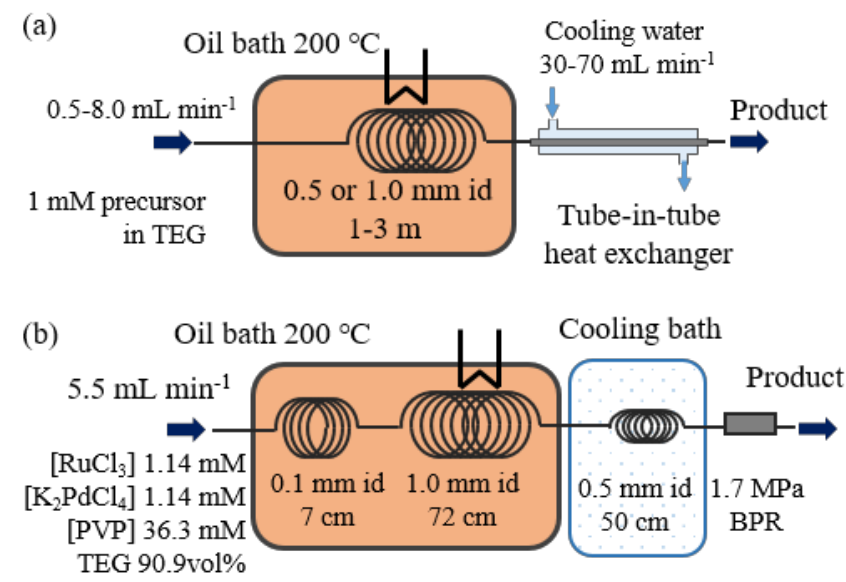

(c)

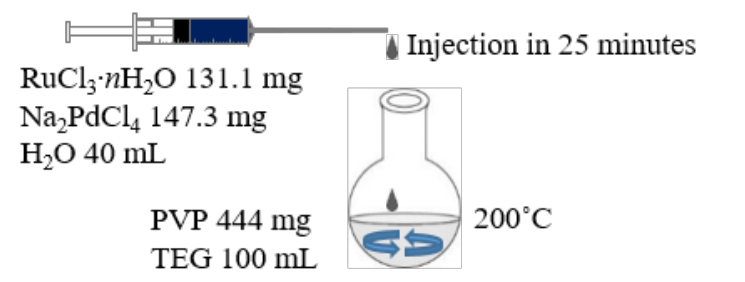

Figure 1. The reactor setup for nanoparticle synthesis: (a) conversion measurement setup with a heat exchanger; (b) rapid heating synthesis with a narrow channel; and (c) a semi-batch system with a round-bottom flask.

Particle characterization. The shape, size, and composition of the particles were analyzed by TEM. TEM images were obtained using a JEOL JEM-2100F instrument. Scanning transmission electron microscopy (STEM) images with energy dispersive X-ray spectroscopy (EDX) were obtained using JEM-2100F and FEI XFEG TITAN systems. High-angle annular dark field (HAADF) detectors were used to acquire the STEM images. The average diameter and standard deviation of the resultant particles were calculated by measuring the area of at least 100 particles 
from the TEM images. XRD patterns were measured with a MultiFlex diffractometer (Rigaku, Tokyo, Japan) using a Cu-K $\alpha(\lambda=1.54184 \AA)$ beam. The atomic composition of the particles was analyzed by ICP-AES. After the XRD analysis, the samples were placed in a high-pressure vessel for metal analysis (SV-25, GL Sciences, Tokyo, Japan) with $4 \mathrm{~mL}$ of aqua regia and then kept at $200^{\circ} \mathrm{C}$ for 1 hour. The dissolved sample was diluted and analyzed for its atomic composition by the ICPS-7500.

\section{RESULTS AND DISCUSSION}

Difference in reduction times between precursors. Figure 2 shows the profiles of the remaining precursor ions that were obtained by utilizing a microflow reactor system. Due to the advantages of the microflow reactor, reaction times as short as $1 \mathrm{~s}$ were successfully examined in our scheme. In the case of $\mathrm{PdCl}_{4}{ }^{2-}$, the remaining precursor in the solution decreased exponentially. Wang et al. ${ }^{15}$ reported that the rate equations of $\mathrm{PdCl}_{4}{ }^{2-}$ reduction could be expressed as quasi first-order reactions. Our results agree with their observations. The reaction was fast and finished in approximately $5 \mathrm{~s}$. In the case of $\mathrm{RuCl}_{3}$, the reaction time was significantly longer than that of $\mathrm{PdCl}_{4}{ }^{2-}$. Even after 4 minutes, $12 \%$ of Ru remained in the solution as ions. The lower reactivity of the Ru precursor than the Pd precursor was also suggested in a recent report by Laura et al., in which Pt-Pd and Pt-Ru bimetallic nanoparticles were synthesized using a microflow reactor ${ }^{36}$. Conversion measurements with the reactant solution dissolving both precursors were also conducted to investigate the interaction between the precursors. It is known that the catalytic cycle works in some combinations of metal precursors and completely changes the reaction rate ${ }^{14}$. Judging from the conversion profiles obtained in the coreduction process, a synergetic reduction reaction between $\mathrm{PdCl}_{4}{ }^{2-}$ and $\mathrm{RuCl}_{3}$ did not occur, suggesting a weak interaction. Kusada et al. surmised that the reduction of $\mathrm{RuCl}_{3}$ was as instantaneous as that of $\mathrm{PdCl}_{4}{ }^{2-}$ at the elevated 
temperature because the color of the precursor solution immediately changed to black. Metal nanoparticle suspensions appear dark, and color is often used as an indicator for successful reduction ${ }^{3}$. Figure 3 shows the color of the obtained $\mathrm{Ru}^{3+}$ solution at the outlet for each residence time. The color changed dramatically in the initial stage, from red to green and then to dark blue. $\mathrm{Ru}^{3+}$ is known to exist in both the hydrolyzed and polymerized forms of the solution ${ }^{35}$. The instant color change results from the numerous reactions of dehydrolyzation, depolymerization, and reduction to $\mathrm{Ru}^{2+}$. A qualitative investigation based on the color of the solution is easy and intuitive but not sufficient for determining the complete conversion, particularly for a multiple reaction system. Consequently, a quantitative evaluation based on the concentration measurements in the reaction solution provides a significant new insight into the underlying mechanism.
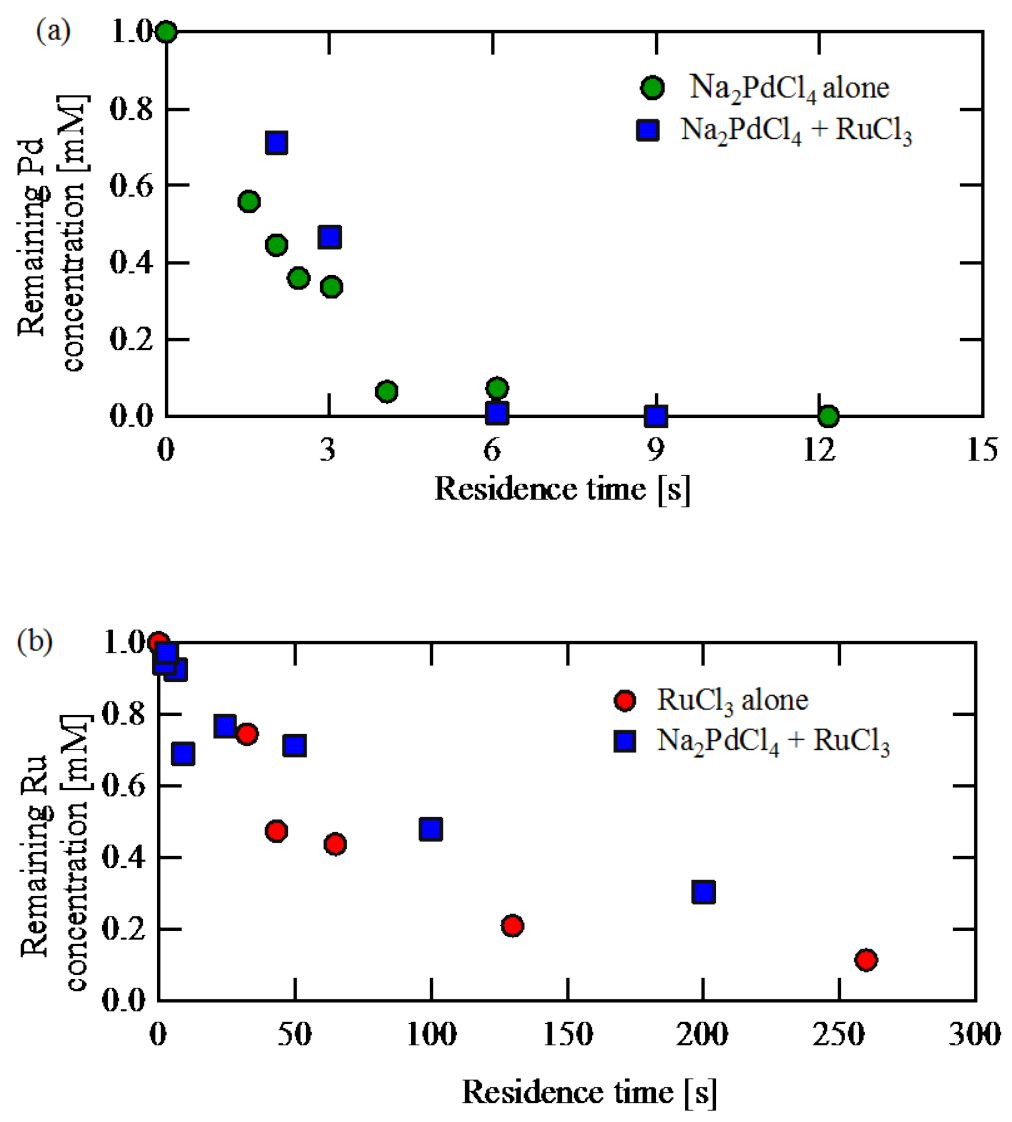
Figure 2. Concentration profiles of the precursor ions reacted at $200^{\circ} \mathrm{C}$ in TEG: (a) $\mathrm{Na}_{2} \mathrm{PdCl}_{4}$ and (b) $\mathrm{RuCl}_{3}$.

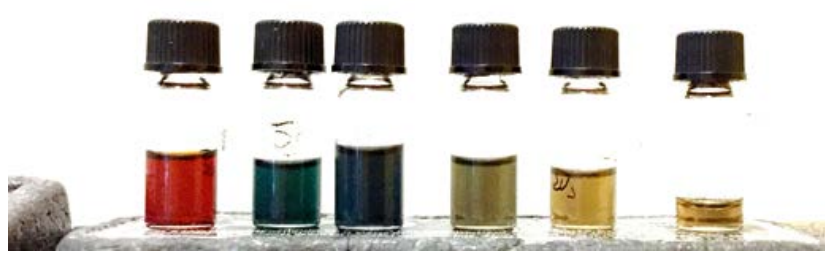

Figure 3. Color changes of the solution containing $\mathrm{RuCl}_{3}$ in TEG: the corresponding residence times at $200^{\circ} \mathrm{C}$ are $0,5,10,30,120$, and $600 \mathrm{~s}$ (from left to right).

Phase segregation with rapid heating. The crucial step for alloying in the semi-batch synthesis at $200^{\circ} \mathrm{C}$ was assumed to be the simultaneous instant reduction of both precursors, enabled by rapid heating during the dropwise injection. However, the slow reduction of $\mathrm{RuCl}_{3}$ described in the previous section contradicts this assumption. With a conventional flask, it is difficult to determine whether the key step for alloying lies in the heating rate or the operation mode itself. Here, we utilized a microflow reactor to instantly heat the precursor and glycol mixture. A premixed solution of the two precursors in TEG was fed into the stainless steel microflow reactor in the oil bath at $200^{\circ} \mathrm{C}$. Figure 4(a) shows an image of the obtained particles. Despite the rapid heating (i.e., approximately $0.3 \mathrm{~s}$ ), there was a clear segregation between larger particles (approximately $10 \mathrm{~nm}$ ) and smaller particles (approximately $3 \mathrm{~nm}$ ). Figure 4(b) shows the highresolution image of the larger particle. This image clearly shows that the particle had a well-defined crystalline structure. Figures 4(c) and (d) provide an elemental analysis of a representative larger particle. The characteristic EDX spectrum of Pd was dominant, with the spectrum of Ru contributing little to the total EDX signal. Thus, it would appear that successful alloying in the semi-batch method is not due to the instant heating of the injected precursor solution. Next, we 
explored the time evolution of bimetallic particles during the semi-batch synthesis to identify the underlying mechanism of alloying.
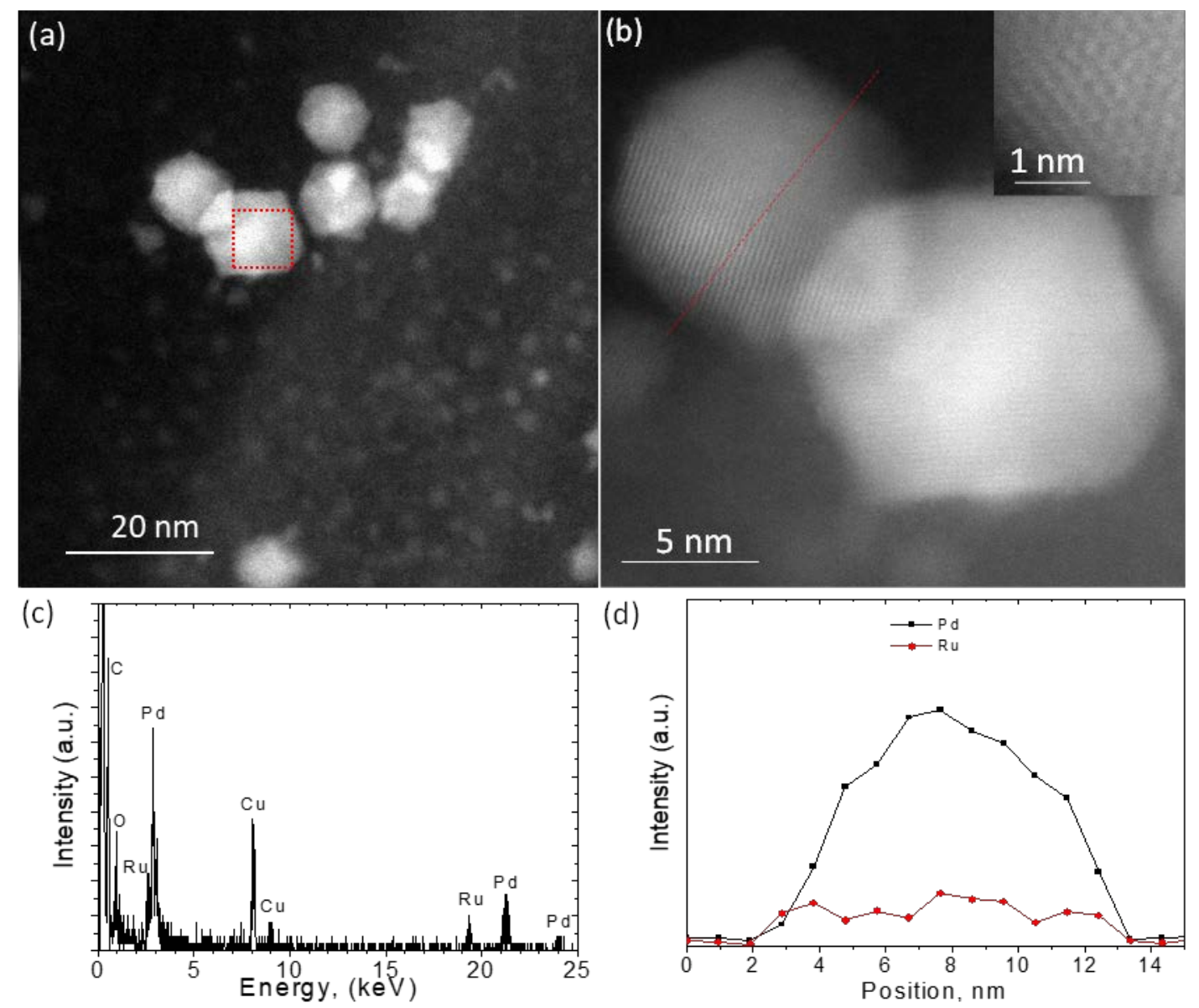

Figure 4. Characterization of the Pd-Ru nanoparticles obtained through the flow synthesis method with rapid heating: (a) An STEM-HAADF image showing segregated particles, (b) a highresolution STEM-HAADF image showing the high crystallinity of the produced Pd-Ru nanoparticles. The inset is a magnification of the image with atomic resolution. (c) EDS analysis at the area marked in Figure 4(a), and (d) Pd and Ru EDS line profiles of the particle in Figure 4(b)

Particle profiles during semi-batch operation. We conducted the original semi-batch experiment by injecting the precursor solution into the heated glycol solution over a 25-minute 
period, followed by 5 hours of aging. Small amounts of the reactant solution were sampled continually and observed using TEM to reveal the progress of the alloy formation. Figure 5(a) presents the results of the STEM-HAADF observation and elemental mapping for each stage of the operation. The particles obtained after the complete procedure aligned with the results of the original report by Kusada et al. The particle size was $12.1 \pm 2.5 \mathrm{~nm}$ (Kusada et al., $10.0 \pm 1.2 \mathrm{~nm}$ ), with homogeneously distributed $\mathrm{Pd}$ and $\mathrm{Ru}$ at the same atomic ratio. The small difference in size could be attributed to the injection time, which was not specified in the original report. At 1 minute of injection, the particles were small, approximately $5 \mathrm{~nm}$, and showed a core-shell-like structure. The Ru signal was low compared with the strong Pd signal. The EDX mapping depicted in Figure 5(a-ii) and (a-iii) indicated that the Pd-Ru nanoparticles became more homogeneous in atomic composition as the reaction time increased. Figure 5(b) summarizes the time evolution of the particle size and the atomic ratio of $\mathrm{Ru}$ to $\mathrm{Pd}$. The semiquantitative data (obtained using a local analysis technique) as the STEM-EDX spectrum and the quantitative data (obtained using a bulk analysis technique) as the ICP-AES results were included. The analysis aligned with the aforementioned results and revealed that the Pd-Ru nanoparticles evolved into particles with large dimensions and that the $\mathrm{Ru} / \mathrm{Pd}$ atomic ratio was increased upon increasing the operation time. However, the STEM-EDX analysis revealed a Ru/Pd value larger than that in the ICP-AES analysis. This mismatch could be attributed to the Ru-rich surfaces at the early stages. The increase in particle size indicates that particle growth was more dominant than nucleation, which would only proceed at the very beginning of the injection. The smaller $\mathrm{Ru} / \mathrm{Pd}$ atomic ratio at the earlier stages is well explained by the slower reduction of $\mathrm{RuCl}_{3}$, as previously discussed. There would be a time delay of several minutes between the injection of $\mathrm{RuCl}_{3}$ and the reduction of $\mathrm{Ru}$ ions to 
solid $\mathrm{Ru}(0)$. The change in the atomic distribution of $\mathrm{Ru}$ in each particle can be explained by an intraparticle solid state diffusion.

(a)
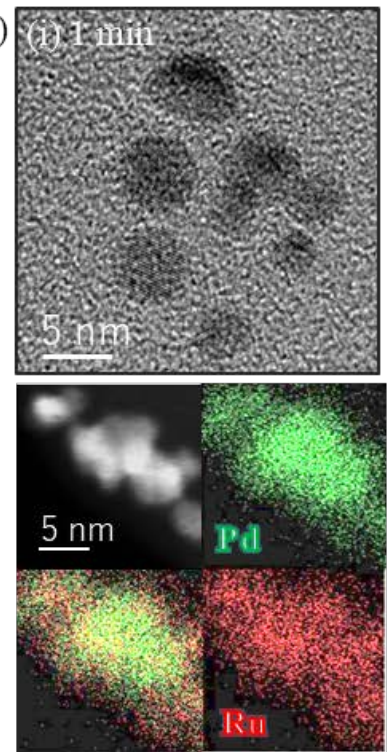
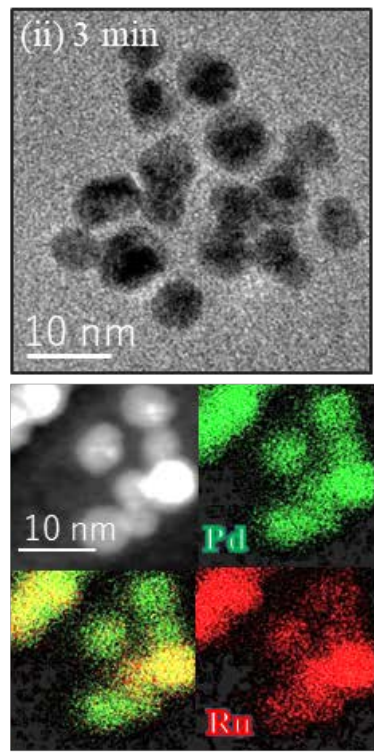
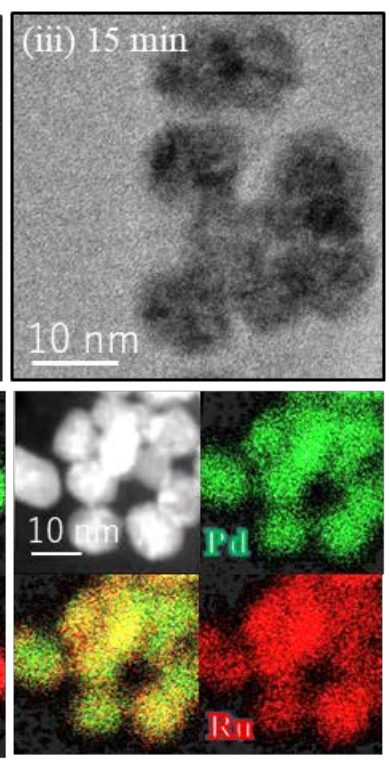
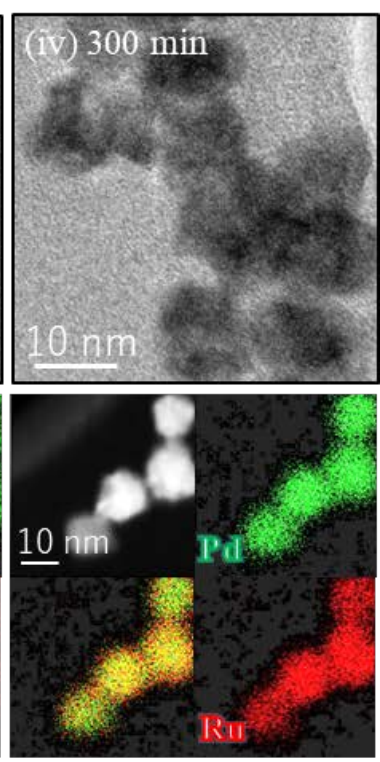
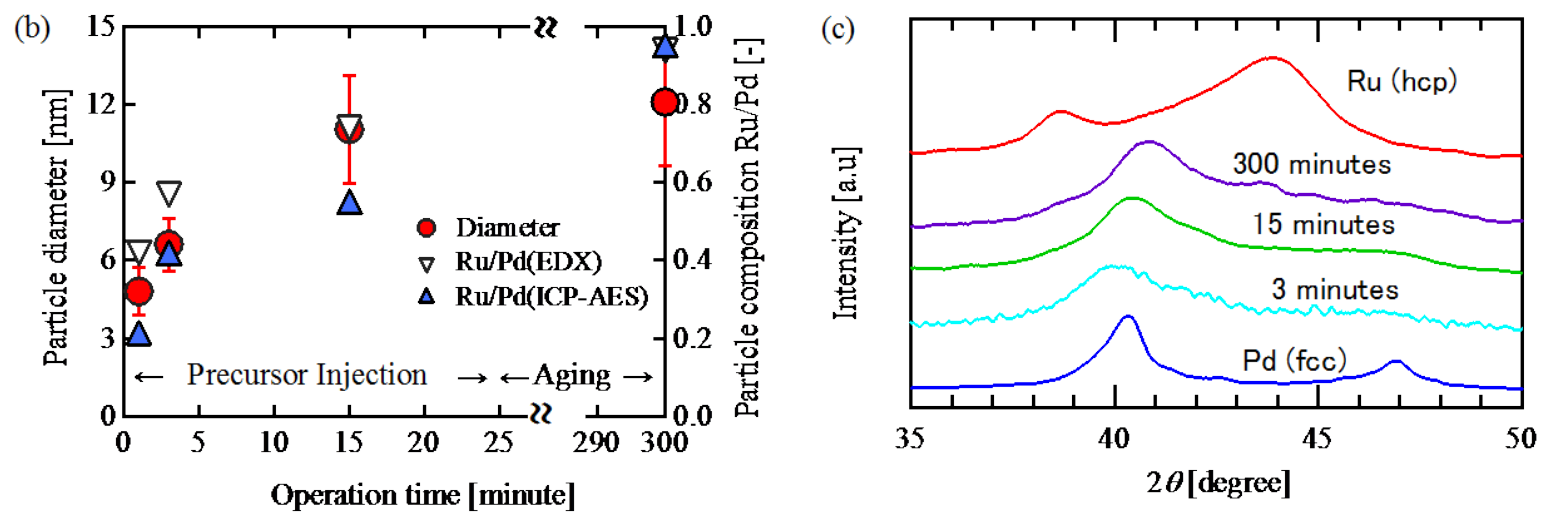

Figure 5. Characterization of Pd-Ru nanoparticles obtained during the semi-batch procedure, with simultaneous addition of precursors: (a) TEM images, STEM-HAADF images, and STEM-EDX mapping of the particles obtained at (i) 1 minute, (ii) 3 minutes, (iii) 15 minutes, and (iv) 300 minutes; (b) the average particle size determined through the TEM images and atomic composition of the nanoparticles; and (c) XRD spectra of the obtained nanoparticles. 
XRD analysis was conducted to clarify the crystallinity and structure of the nanoparticles. Figure 5(c) shows the XRD spectra of the Pd-Ru samples and the reference monometallic nanoparticles. The XRD spectrum of the 300-minute reaction was confirmed to be identical to the spectrum of Pd-Ru alloyed nanoparticles reported in the original literature ${ }^{31}$. The comparison is included in the Supporting Information (Figure S1). Pd and Ru have a similar atomic radius; however, they have a different coordination at the crystal unit cell, with one having a face-centered cubic (fcc) structure and the other having a hexagonal close-packed (hcp) structure. In a previous study, Pd-Ru alloyed nanoparticles were confirmed to have both fcc and hcp structures in a single solid solution nanoparticle through synchrotron XRD analysis and selected area diffraction pattern TEM analysis $^{31}$. The final products at 300 minutes would have the same fcc and hcp structures in a single particle. Judging from the observed spectra shift during the semi-batch procedure, some change in the structure, such as the evolution of crystalline arrangement or structural relaxation, would have occurred with the solid state diffusion. A well-defined fcc phase was observed at a short synthesis time, and an hcp phase was weakly observed as the reaction time was increased (Figure 5-c). These results align with those in the literature ${ }^{37}$, in which the lattice parameter of the alloyed Pd-Ru nanoparticles was increased linearly when the Pd atomic ratio was increased by changing the concentration of the Pd precursor, indicating a trend of lattice relaxation ${ }^{37}$.

Requirements for successful solid state diffusion. Wu et al. synthesized sub-5 nm Pd-Ru alloyed nanoparticles using another procedure ${ }^{34}$. In their method, a $\mathrm{RuCl}_{3}$ solution was first added to EG at $196^{\circ} \mathrm{C}$ to synthesize $\mathrm{Ru}$ seeds. Second, a $\mathrm{K}_{2} \mathrm{PdCl}_{4}$ solution was introduced dropwise for approximately 4-5 minutes to deposit Pd atoms on the seeds. Then, the mixture was refluxed for 1 hour so that the solid state diffusion could be completed. The simultaneous precursor introduction method by Kusada et al. and the sequential introduction method by Wang et al. were considered 
to have different alloying mechanisms ${ }^{38}$. However, our results suggest that the solid state diffusion of the later reduced and deposited atoms on the seeds is a common and critical mechanism for realizing alloyed nanoparticles in the Ru-Pd system. We conducted the sequential addition of precursors at $200^{\circ} \mathrm{C}$ in TEG to clarify the prerequisites of successful solid state diffusion. Figures 6(a-i) and (a-ii) show the TEM images of the seeds synthesized by introducing one precursor into the heated TEG. Ru resulted in seeds measuring $4.1 \pm 0.6 \mathrm{~nm}$ in diameter, and Pd formed more regular structures of truncated octahedrons, with a diameter of $11.6 \pm 2.3 \mathrm{~nm}$. Figures 6(a-iii) and (a-iv) show an STEM-EDX mapping of the Pd-Ru bimetallic nanoparticles that were obtained after introducing another metal precursor to the seeds. In both cases, the bimetallic nanoparticles resulted in core-shell structures in which the seed metal occupied the core and another metal existed in the shell. These results indicated that presynthesized cores were too rigid for surface atoms to diffuse into and that a high temperature alone is not enough for successful solid state diffusion. Figure 6(b) presents the XRD spectra of the Pd-Ru nanoparticles obtained through the sequential addition of precursors. The spectra of seeds and alloyed Pd-Ru nanoparticles are also shown as a reference. Pd precursor injection into the Ru seeds resulted in the spectrum that had all the characteristic peaks of monometallic Pd and Ru. Pd would have formed fcc crystalline structures separately from the Ru seeds. Ru precursor injection into the Pd seeds resulted in a spectrum that was similar to but slightly different from the Pd seeds. In both cases, the sequential addition of precursors resulted in a crystalline structure that was completely different from the one formed by the alloyed nanoparticles obtained through the simultaneous addition. Alloying by galvanic replacement, which is driven by the difference in redox potentials and plays an important role in some systems ${ }^{39,40}$, was not confirmed in this study. 
(a)
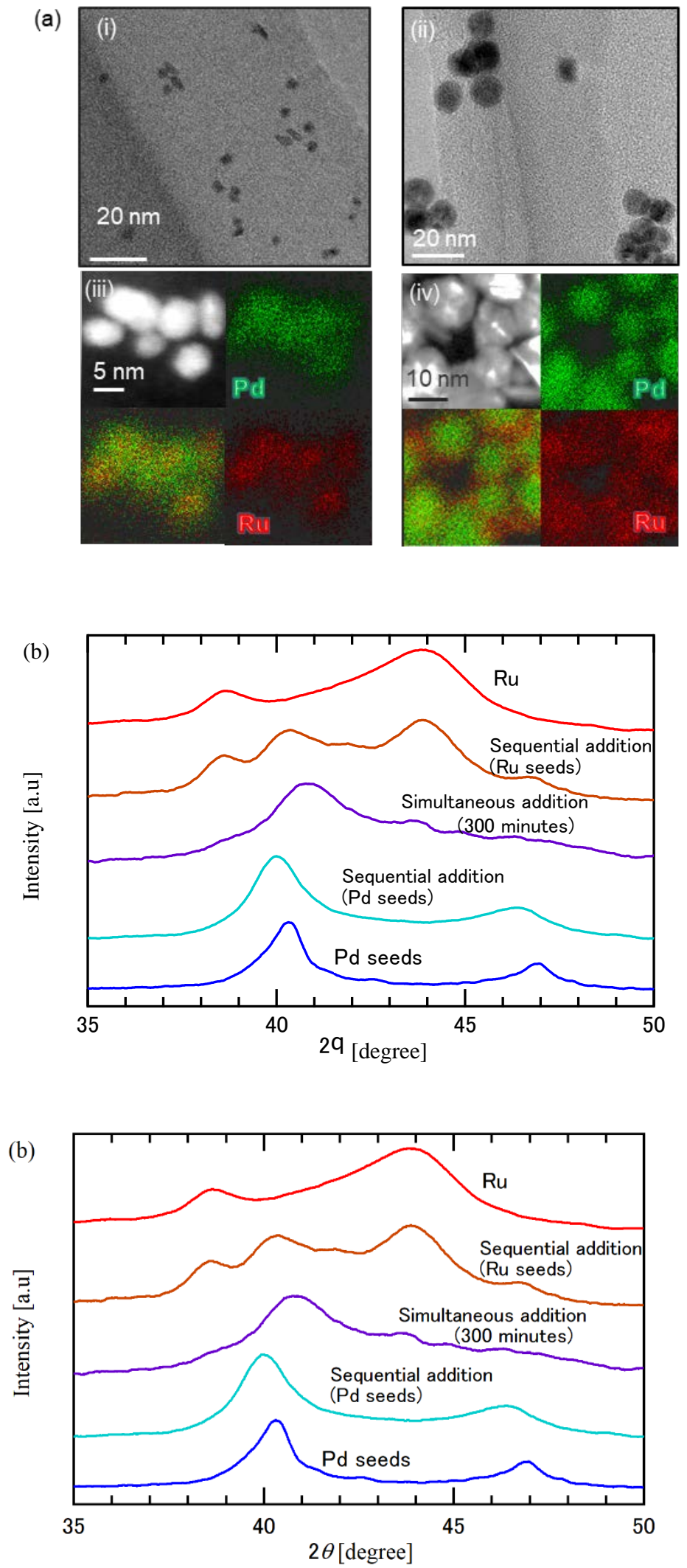
Figure 6. Characterization of Pd-Ru nanoparticles obtained through the sequential addition of each precursor: (a-i) TEM image of Ru seeds synthesized by a 20-minute injection, (a-ii) TEM image of Pd seeds synthesized by a 20-minute injection, (a-iii) STEM-HAADF images and STEM-EDX mapping of Pd-Ru nanoparticles obtained after the injection of the Pd precursor into Ru seeds, (aiv) STEM-HAADF images and STEM-EDX mapping of Pd-Ru nanoparticles obtained after the injection of the Ru precursor into Pd seeds, and (b) XRD spectra of the obtained nanoparticles.

Figure 7 shows a model of the mechanism for forming Pd-Ru alloyed nanoparticles driven by solid state diffusion, according to the results obtained in this report. First, the dropwise injection of the precursor solution keeps the precursor concentration low in the reacting media. Then, the Pd precursor is reduced in seconds and nucleates to form seeds with low crystallinity. It is well known that a small supersaturation tends to cause stacking faults, dislocations, or twin defects during the nucleation stage ${ }^{15,41}$. If all precursors are dissolved in glycol and then heated, Pd immediately forms crystalline particles because of the high supersaturation, resulting in phase segregation, as shown in Figure 4. If seeds with low crystallinity can be obtained, then the sequential addition of precursors is possible, as described in the method by Wu et al $l^{34}$. Next, the subsequent continuous supply and reduction of precursors enable the deposition of reduced atoms on the particle surface. The low supersaturation suppresses further homogeneous nucleation. Surface atoms then diffuse into the particle to form an alloyed nanoparticle with the assistance of the high temperature used in the polyol reducing method. If the seeds have a rigid crystal structure, then the surface atoms fail to enter into the seeds and form a shell, as shown in Figures 6(a-iii) and (a-iv). 


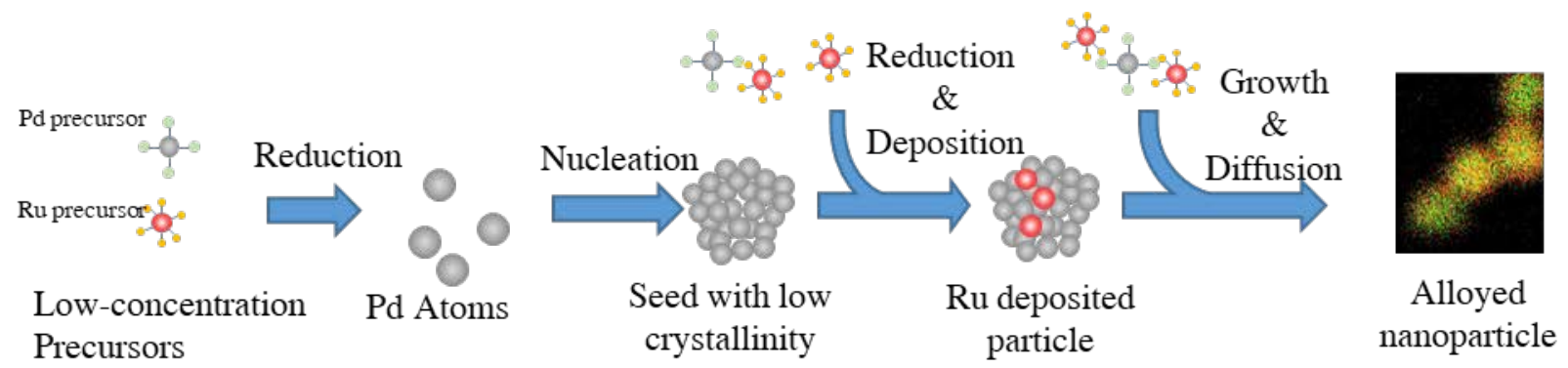

Figure 7. A model of the Pd-Ru alloy formation mechanism driven by solid state diffusion.

From the above revealed mechanism, we propose the following guidelines for designing a new synthetic procedure that is aimed at the controlled production of alloyed $\mathrm{Pd}-\mathrm{Ru}$ nanostructures: 1) Start by making seeds with low crystallinity to prevent the formation of a rigid monometallic crystal structure. 2) Maintain a low supersaturation for a long period to undergo the deposition and diffusion cycle while avoiding homogeneous nucleation. 3) Operate at an elevated temperature by using the polyol and solvothermal processes for promoting solid state diffusion.

These guidelines may be general and of great interest for the production of alloyed nanoparticles where a variety of combinations of elements is required.

\section{CONCLUSIONS}

In this study, a mechanistic study of the formation of alloyed Pd-Ru nanoparticles was performed through conversion measurements, flow synthesis, and semi-batch synthesis with sampling over time. Microflow reactors were utilized for their rapid heating ability and controlled short residence 
time. Microflow reactors enabled a quantitative investigation of the reducing time and interaction between precursors. Our approach revealed a much slower reducing time for $\mathrm{RuCl}_{3}$ than for $\mathrm{Na}_{2} \mathrm{PdCl}_{4}$ and demonstrated that only rapid heating of the reactant solution causes phase segregation in particles. We propose a solid state diffusion mechanism for Pd-Ru alloyed nanoparticle synthesis, with further investigation of the semi-batch method. Quantitative and reliable conversion measurements under harsh conditions utilizing a microflow reactor could be a powerful tool to expand our understanding of the complex phenomena involved in nanoparticle synthesis. 


\section{ASSOCIATED CONTENT}

\section{Supporting Information.}

The following file is available free of charge.

Calculation of heating time in the microflow reactor, comparison of the XRD spectra, and particle size distribution obtained through the semi-batch operation (PDF).

\section{AUTHOR INFORMATION}

\section{Corresponding Author}

*Email: shusaku_asano@cm.kyushu-u.ac.jp

\section{Present Address}

$\diamond$ Institute for Materials Chemistry and Engineering, Kyushu University, 816-8580 Kasuga, Japan

\section{Notes}

The authors have declared no competing financial interest.

\section{ACKNOWLEDGMENT}

This work was supported by the Japan Society for the Promotion of Science (JSPS), grant numbers 25220913 and 15J00287. S.A. was supported by a graduate exchange fellowship from JGP, Kyoto University. A part of this work was supported by the Kyoto University Nano Technology Hub in the "Nanotechnology Platform Project", sponsored by the Ministry of Education, Culture, Sports, Science and Technology (MEXT), Japan. 


\section{REFERENCES}

(1) Xia, Y.; Gilroy, K. D.; Peng, H.-C.; Xia, X. Seed-Mediated Growth of Colloidal Metal Nanocrystals. Angew. Chemie Int. Ed. 2017, 56 (1), 60-95.

https://doi.org/10.1002/anie.201604731.

(2) Zhou, S.; Jackson, G. S.; Eichhorn, B. AuPt Alloy Nanoparticles for CO-Tolerant Hydrogen Activation: Architectural Effects in Au-Pt Bimetallic Nanocatalysts. Adv.

Funct. Mater. 2007, 17 (16), 3099-3104. https://doi.org/10.1002/adfm.200700216.

(3) Alayoglu, S.; Eichhorn, B. Rh-Pt Bimetallic Catalysts: Synthesis, Characterization, and Catalysis of Core-Shell, Alloy, and Monometallic Nanoparticles. J. Am. Chem. Soc. 2008, 130 (51), 17479-17486. https://doi.org/10.1021/ja8061425.

(4) Shan, S.; Luo, J.; Yang, L.; Zhong, C.-J. Nanoalloy Catalysts: Structural and Catalytic Properties. Catal. Sci. Technol. 2014, 4 (10), 3570-3588. https://doi.org/10.1039/C4CY00469H.

(5) Alayoglu, S.; Nilekar, A. U.; Mavrikakis, M.; Eichhorn, B. Ru-Pt Core-shell Nanoparticles for Preferential Oxidation of Carbon Monoxide in Hydrogen. Nat. Mater. 2008, 7 (4), 333-338. https://doi.org/10.1038/nmat2156.

(6) Kattel, S.; Ramírez, P. J.; Chen, J. G.; Rodriguez, J. A.; Liu, P. Active Sites for CO 2 Hydrogenation to Methanol on Cu/ZnO Catalysts. Science (80-. ). 2017, 355 (6331), 1296-1299. https://doi.org/10.1126/science.aal3573. 
(7) Jukk, K.; Kongi, N.; Tammeveski, K.; Solla-Gullón, J.; Feliu, J. M. PdPt Alloy Nanocubes as Electrocatalysts for Oxygen Reduction Reaction in Acid Media. Electrochem. commun. 2015, 56, 11-15. https://doi.org/10.1016/j.elecom.2015.04.001.

(8) Usón, L.; Sebastian, V.; Mayoral, A.; Hueso, J. L.; Eguizabal, A.; Arruebo, M.; Santamaria, J. Spontaneous Formation of Au-Pt Alloyed Nanoparticles Using Pure NanoCounterparts as Starters: A Ligand and Size Dependent Process. Nanoscale 2015, 7 (22), 10152-10161. https://doi.org/10.1039/C5NR01819F.

(9) Wang, Q.; Li, B.; Zhou, X.; Liu, J.; Hu, J. Controlling Shape and Plasmon Resonance of Pt- Etched Au @ Ag Nanorods. Langmuir 2018, 34, 5719-5727. https://doi.org/10.1021/acs.langmuir.8b00328.

(10) Xu, Y.; Chen, L.; Wang, X.; Yao, W.; Zhang, Q. Recent Advances in Noble Metal Based Composite Nanocatalysts: Colloidal Synthesis, Properties, and Catalytic Applications. Nanoscale 2015, 7 (24), 10559-10583. https://doi.org/10.1039/c5nr02216a.

(11) Zhang, H.; Jin, M.; Xia, Y. Enhancing the Catalytic and Electrocatalytic Properties of PtBased Catalysts by Forming Bimetallic Nanocrystals with Pd. Chem Soc Rev 2012, 41 (24), 8035-8049. https://doi.org/10.1039/c2cs35173k.

(12) Hueso, J. L.; Sebastián, V.; Mayoral, Á.; Usón, L.; Arruebo, M.; Santamaría, J. Beyond Gold: Rediscovering Tetrakis-(Hydroxymethyl)-Phosphonium Chloride (THPC) as an Effective Agent for the Synthesis of Ultra-Small Noble Metal Nanoparticles and PtContaining Nanoalloys. RSC Adv. 2013, 3 (26), 10427. https://doi.org/10.1039/c3ra40774h. 
(13) Sano, N.; Nakanishi, Y.; Sugiura, K.; Yamanaka, H.; Tamon, H.; Saito, N.; Konishi, Y. Synthesis of Bimetallic Pt-Ru Nanoparticles by Bioreduction Using Shewanella Algae for Application to Direct Methanol Fuel Cell. J. Chem. Eng. Japan 2016, 49 (5), 488-492. https://doi.org/10.1252/jcej.15we077.

(14) Yonezawa, T.; Toshima, N. Mechanistic Consideration of Formation of PolymerProtected Nanoscopic Bimetallic Clusters. J. Chem. Soc. Faraday Trans. 1995, 91 (22), 4111. https://doi.org/10.1039/ft9959104111.

(15) Wang, Y.; Peng, H. C.; Liu, J.; Huang, C. Z.; Xia, Y. Use of Reduction Rate as a Quantitative Knob for Controlling the Twin Structure and Shape of Palladium Nanocrystals. Nano Lett. 2015, 15 (2), 1445-1450. https://doi.org/10.1021/acs.nanolett.5b00158.

(16) Mae, K. Advanced Chemical Processing Using Microspace. Chem. Eng. Sci. 2007, 62 (18-20), 4842-4851. https://doi.org/10.1016/j.ces.2007.01.012.

(17) Tofighi, G.; Gaur, A.; Doronkin, D. E.; Lichtenberg, H.; Wang, W.; Wang, D.; Rinke, G.; Ewinger, A.; Dittmeyer, R.; Grunwaldt, J. D. Microfluidic Synthesis of Ultrasmall AuPd Nanoparticles with a Homogeneously Mixed Alloy Structure in Fast Continuous Flow for Catalytic Applications. J. Phys. Chem. C 2018, 122 (3), 1721-1731. https://doi.org/10.1021/acs.jpcc.7b11383.

(18) Tofighi, G.; Lichtenberg, H.; Pesek, J.; Sheppard, T. L.; Wang, W.; Schöttner, L.; Rinke, G.; Dittmeyer, R.; Grunwaldt, J.-D. Continuous Microfluidic Synthesis of Colloidal Ultrasmall Gold Nanoparticles: In Situ Study of the Early Reaction Stages and 
Application for Catalysis. React. Chem. Eng. 2017, 2 (6), 876-884.

https://doi.org/10.1039/C7RE00114B.

(19) Sebastián, V.; Jensen, K. F. Nanoengineering a Library of Metallic Nanostructures Using a Single Microfluidic Reactor. Nanoscale 2016, 8 (33), 15288-15295. https://doi.org/10.1039/C6NR04104C.

(20) Marre, S.; Park, J.; Rempel, J.; Guan, J.; Bawendi, M. G.; Jensen, K. F. Supercritical Continuous-Microflow Synthesis of Narrow Size Distribution Quantum Dots. Adv. Mater. 2008, 20 (24), 4830-4834. https://doi.org/10.1002/adma.200801579.

(21) Sebastian, V.; Smith, C. D.; Jensen, K. F. Shape-Controlled Continuous Synthesis of Metal Nanostructures. Nanoscale 2016, 7534-7543. https://doi.org/10.1039/C5NR08531D.

(22) Asano, S.; Yamada, S.; Maki, T.; Muranaka, Y.; Mae, K. Design Protocol of Microjet Mixers for Achieving Desirable Mixing Times with Arbitrary Flow Rate Ratios. React. Chem. Eng. 2017, 2 (6), 830-841. https://doi.org/10.1039/C7RE00051K.

(23) Mandal, M. M.; Serra, C.; Hoarau, Y.; Nigam, K. D. P. Numerical Modeling of Polystyrene Synthesis in Coiled Flow Inverter. Microfluid. Nanofluidics 2011, 10 (2), 415-423. https://doi.org/10.1007/s10404-010-0679-z.

(24) Hohmann, L.; Greinert, T.; Mierka, O.; Turek, S.; Schembecker, G.; Bayraktar, E.; Wohlgemuth, K.; Kockmann, N. Analysis of Crystal Size Dispersion Effects in a Continuous Coiled Tubular Crystallizer: Experiments and Modeling. Cryst. Growth Des. 2018, acs.cgd.7b01383. https://doi.org/10.1021/acs.cgd.7b01383. 
(25) Singh, J.; Kockmann, N.; Nigam, K. D. P. Novel Three-Dimensional Microfluidic Device for Process Intensification. Chem. Eng. Process. Process Intensif. 2014, 86, 78-89. https://doi.org/10.1016/j.cep.2014.10.013.

(26) Sultana, M.; Jensen, K. F. Microfluidic Continuous Seeded Crystallization: Extraction of Growth Kinetics and Impact of Impurity on Morphology. Cryst. Growth Des. 2012, 12 (12), 6260-6266. https://doi.org/10.1021/cg301538y.

(27) Xie, L.; Shen, Y.; Franke, D.; Sebastián, V.; Bawendi, M. G.; Jensen, K. F. Characterization of Indium Phosphide Quantum Dot Growth Intermediates Using MALDI-TOF Mass Spectrometry. J. Am. Chem. Soc. 2016, 138 (41), 13469-13472. https://doi.org/10.1021/jacs.6b06468.

(28) Seibt, S.; With, S.; Bernet, A.; Schmidt, H.-W.; Förster, S. Hydrogelation Kinetics Measured in a Microfluidic Device with In-Situ X-Ray and Fluorescence Detection. Langmuir 2018, acs.langmuir.8b00384. https://doi.org/10.1021/acs.langmuir.8b00384.

(29) Zhang, H.; Lakerveld, R.; Heider, P. L.; Tao, M.; Su, M.; Testa, C. J.; Dantonio, A. N.; Barton, P. I.; Braatz, R. D.; Trout, B. L.; et al. Application of Continuous Crystallization in an Integrated Continuous Pharmaceutical Pilot Plant. Cryst. Growth Des. 2014, 14 (5), 2148-2157. https://doi.org/10.1021/cg401571h.

(30) Tripathi, S. N.; Bharadwaj, S. R.; Dharwadkar, S. R. The Pd-Ru System (PalladiumRuthenium). J. Phase Equilibria 1993, 14 (5), 638-642. https://doi.org/10.1007/BF02669157. 
(31) Kusada, K.; Kobayashi, H.; Ikeda, R.; Kubota, Y.; Takata, M.; Toh, S.; Yamamoto, T.; Matsumura, S.; Sumi, N.; Sato, K.; et al. Solid Solution Alloy Nanoparticles of Immiscible Pd and Ru Elements Neighboring on Rh: Changeover of the Thermodynamic Behavior for Hydrogen Storage and Enhanced Co-Oxidizing Ability. J. Am. Chem. Soc. 2014, 136 (5), 1864-1871. https://doi.org/10.1021/ja409464g.

(32) Kutubi, M. S.; Sato, K.; Wada, K.; Yamamoto, T.; Matsumura, S.; Kusada, K.; Kobayashi, H.; Kitagawa, H.; Nagaoka, K. Dual Lewis Acidic/Basic Pd0.5Ru0.5-Poly(NVinyl-2-Pyrrolidone) Alloyed Nanoparticle: Outstanding Catalytic Activity and Selectivity in Suzuki-Miyaura Cross-Coupling Reaction. ChemCatChem 2015, 7 (23), 3887-3894. https://doi.org/10.1002/cctc.201500758.

(33) Sato, K.; Tomonaga, H.; Yamamoto, T.; Matsumura, S.; Zulkifli, N. D. B.; Ishimoto, T.; Koyama, M.; Kusada, K.; Kobayashi, H.; Kitagawa, H.; et al. A Synthetic Pseudo-Rh: NOx Reduction Activity and Electronic Structure of Pd-Ru Solid-Solution Alloy Nanoparticles. Sci. Rep. 2016, 6 (1), 28265. https://doi.org/10.1038/srep28265.

(34) Wu, D.; Cao, M.; Shen, M.; Cao, R. Sub-5 Nm Pd-Ru Nanoparticle Alloys as Efficient Catalysts for Formic Acid Electrooxidation. ChemCatChem 2014, 6 (6), 1731-1736. https://doi.org/10.1002/cctc.201400086.

(35) Rich, R. Inorganic Reactions in Water; Springer Berlin Heidelberg: Berlin, Heidelberg, 2007. https://doi.org/10.1007/978-3-540-73962-3. 
(36) Laura, U.; Arruebo, M.; Sebastian, V. Towards the Continuous Production of Pt-Based Heterogeneous Catalysts Using Microfluidic Systems. Dalt. Trans. 2018, 47 (5), 16931702. https://doi.org/10.1039/C7DT03360E.

(37) Miao, K.; Luo, Y.; Zou, J.; Yang, J.; Zhang, F.; Huang, L.; Huang, J.; Kang, X.; Chen, S. PdRu Alloy Nanoparticles of Solid Solution in Atomic Scale: Outperformance towards Formic Acid Electro-Oxidation in Acidic Medium. Electrochim. Acta 2017, 251, 588594. https://doi.org/10.1016/j.electacta.2017.08.167.

(38) Wu, D.; Kusada, K.; Kitagawa, H. Recent Progress in the Structure Control of Pd-Ru Bimetallic Nanomaterials. Sci. Technol. Adv. Mater. 2016, 17 (1), 583-596. https://doi.org/10.1080/14686996.2016.1221727.

(39) Yang, P.; Yuan, X.; Hu, H.; Liu, Y.; Zheng, H.; Yang, D.; Chen, L.; Cao, M.; Xu, Y.; Min, Y.; et al. Solvothermal Synthesis of Alloyed Ptni Colloidal Nanocrystal Clusters (CNCs) with Enhanced Catalytic Activity for Methanol Oxidation. Adv. Funct. Mater. 2018, 28 (1), 1-8. https://doi.org/10.1002/adfm.201704774.

(40) Kim, N. R.; Shin, K.; Jung, I.; Shim, M.; Lee, H. M. Ag-Cu Bimetallic Nanoparticles with Enhanced Resistance to Oxidation: A Combined Experimental and Theoretical Study. J. Phys. Chem. C 2014, 118 (45), 26324-26331. https://doi.org/10.1021/jp506069c.

(41) Xia, Y.; Xiong, Y.; Lim, B.; Skrabalak, S. E. Shape-Controlled Synthesis of Metal Nanocrystals: Simple Chemistry Meets Complex Physics? Angew. Chemie Int. Ed. 2009, 48 (1), 60-103. https://doi.org/10.1002/anie.200802248. 
TOC GRAPHIC

For Table of Contents Only.

Conversion Profiles under a Harsh Condition

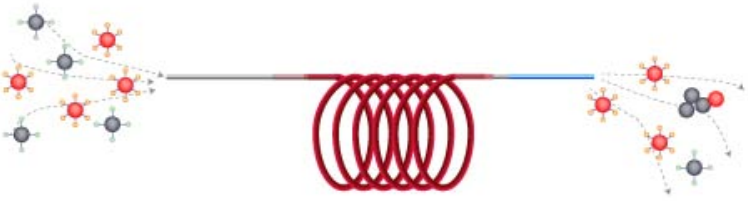

Seconds to minutes at $200^{\circ} \mathrm{C}$ 